\title{
Atezolizumab and Pembrolizumab in Triple-Negative Breast Cancer: A Meta-Analysis
}

\author{
Mahwish Amin'1, Shaiza Sharif2², Waleed Asghar ${ }^{3}$, Humaira Sadaf $^{4}$, Azza Sarfraz $^{5}$, Zouina Sarfraz ${ }^{6}$, Ivan Cherrez- \\ Ojeda $^{7}$
}

\author{
Sir Ganga Ram Hospital Lahore, Punjab, Pakistan; wish0amin94@gmail.com \\ Women Medical Officer, RHC Aroti, PHFMC, Pakistan; shizsharif@gmail.com \\ City Gynae Hospital Toba Tek Singh, Pakistan; waleed0298@gmail.com \\ 4 University of Texas Health Science Centers, McGovern Medical School, Houston, Texas, USA; do- \\ chumairasadaf@gmail.com \\ 5 Aga Khan University, Karachi, Sindh, Pakistan; azza.sarfraz@aku.edu \\ 6 Fatima Jinnah Medical University, Lahore, Punjab, Pakistan; zouinasarfraz@gmail.com \\ 7 Universidad Espíritu Santo, Samborondón, Ecuador; ivancherrez@gmail.com \\ * Correspondence: Dr. Azza Sarfraz; azza.sarfraz@aku.edu; Tel: 0092-333-872-7011
}

\begin{abstract}
Triple negative breast cancer (TNBC) has a higher mRNA expression of programmed cell death ligand 1 (PD-L1) which is a ligand to programmed cell death protein 1 (PD-1). The binding of the ligand leads to suppressed activity of T-cell-mediated immune response against cancer cells. The approval of anti-PD-L1 drugs including pembrolizumab and atezolizumab in subgroups of TNBC offer potential improvement to the current treatment regimens available for TNBC. We conducted a meta-analysis to review the efficacy of pembrolizumab and atezolizumab for the treatment of TNBC in both adjuvant and neo-adjuvant settings. A systematic strategy was used as per the PRISMA 2020 statement. All statistical analyses were conducted using Review Manager 5.4. Outcome measures included objective response rate, progression free survival, overall survival in adjuvant therapy groups, and pathological complete response rates in neoadjuvant groups. Six clinical trials were included. For adjuvant therapies, the ORR (OR=1.26, P =0.04) of Atezolizumab/Pembrolizumab plus chemotherapy was higher in intention to treat (ITT) arms than the placebo groups in TNBC. A positive effect size was found for PFS in the ITT arms (Cohen's $d=1.55, P<0.001)$. The Atezolizumab plus chemotherapy group had a positive effect size for OS compared to the control groups (Cohen's $\mathrm{d}=0.52, \mathrm{P}<0.001$ ). In the neo-adjuvant setting, patients in ITT arms had higher $\mathrm{pCR}$ rates as compared to the control groups $(\mathrm{OR}=1.61, \mathrm{P}=0.001)$. Our findings collate evidence of pembrolizumab and atezolizumab as a viable treatment option among patients with TNBC with PDL1+ subgroups deriving benefits.
\end{abstract}

Keywords: triple negative breast cancer; Pembrolizumab; Atezolizumab; chemotherapy; anti-PDL1; biomarkers; targeted therapies; development of novel drugs

\section{Introduction}

Triple-negative breast cancer (TNBC) is an aggressive type of breast cancer [1]. TNBC is associated with high rates of recurrence and worse prognosis than other breast cancer subtypes. Until recently, cytotoxic chemotherapy had been the mainstay of TNBC management [2]. However, the efficacy of immunotherapy in triple-negative breast cancer remains unclear. Targeted immunotherapy is currently underway as a viable treatment option for various cancers including more aggressive breast cancers such as TNBC. The binding of the ligand PD-L1 to PD-1 results in the suppressed activity of the T-cell-mediated immune response against cancer cells [3]. TNBC has a higher programmed cell death ligand 1 (PD-L1) mRNA expression which is a ligand to programmed cell death protein 1 
(PD-1) [3]. The United States Food and Drug Administration (U.S. FDA) has approved two checkpoint inhibitors for TNBC including atezolizumab in combination with nabpaclitaxel and pembrolizumab in combination with chemotherapy for PD-L1+ advanced TNBC in March 2019 [4], and November 2020 [5], respectively. Pembrolizumab is a highly selective humanized monoclonal IgG-4 antibody that blocks the PD-1 receptor on the cell surface, resulting in activation of T-cell mediated immune responses against tumor cells similar to atezolizumab [6]. Atezolizumab is another highly-selective humanized IgG-1 monoclonal antibody that binds to the ligand of PD-1 known as PD-L1, thereby blocking its interaction with PD-1, and resulting in enhanced T-cell activity against tumor cells [6].

The following meta-analysis synthesizes common endpoints of atezolizumab and Pembrolizumab clinical trials among patients with TNBC. Clinical trials enrolling patients with any stage of TNBC, receiving any of the two aforementioned immunotherapeutic agents, and as first-line or beyond, will be statistically interpreted to present a quantitative summary of available data. The aim of the following paper is to comment on the efficacy of immune checkpoint inhibitors atezolizumab and pembrolizumab in adjuvant and neoadjuvant settings among patients with TNBC. While cautiousness is required, our findings are an updated guide to current practices, comprising of recent literature, addressing the efficacy and safety of these agents for PD-1/PD-L1 positive, early-stage, locally advanced, and metastatic TNBC.

\section{Objective}

The objective is to assess the efficacy of immune checkpoint inhibitors including atezolizumab and pembrolizumab among the entire group and PD-L1 positive subgroups, measured as pathological complete response ( $\mathrm{pCR}$ ) in neoadjuvant settings, and progressionfree survival (PFS), objective response rate (ORR), and overall survival (OS) in adjuvant settings.

\section{Methods}

Using the Preferred Reporting Items for Systematic Reviews and Meta-Analyses (PRISMA), statement 2020, clinical trials were included in the meta-analysis that reported evidence of efficacy or safety in TNBC [7]. Specific outcomes were reported for PD-L1 positive and negative sub-groups. When applicable, the control group, receiving placebo, was also compared with the intention-to-treat (ITT) group. The meta-analytical findings present pertinent evidence for current TNBC care, while comparing to controlled groups, for clinical trials of immune checkpoint inhibitors (atezolizumab and pembrolizumab) approved in triple-negative breast cancer (TNBC).

\subsection{Inclusion and Exclusion Criteria}

The following type of studies were included: randomized and/or controlled clinical trials. All cohort studies, case-controlled studies, and case series were excluded due to the high risk of associated biases. High-quality clinical trials that compared PD-L1 positive and negative sub-groups were included. All other trials and entries that did not focus on these endpoints were excluded. We included studies of adult participants regardless of their age groups. A follow-up time period was not pre-determined for inclusion due to the dearth of data. The target condition was triple-negative breast cancer with further exploration of PD-L1 expression.

\subsection{Study Tasks}

Included trials investigated efficacy (using different markers) endpoints. These were further tabulated by all researchers in a shared spreadsheet, discussed as baseline characteristics (trial's name, phase, design, total sample (n), treatment, target population) and outcome measures (objective response rate (ORR), progression free survival (PFS), 
and overall survival (OS) in adjuvant therapy groups; and pathological complete response (pCR) rates in neoadjuvant groups) in total and PDL1+ subgroups.

\subsection{Comparator/Control}

Outcome measures in individuals with triple-negative breast cancer were compared to the controlled/placebo arm in clinical trials with multiple arms.

\subsection{Search Strategy}

A unique, systematic search strategy was used to search key electronic databases, registers and other sources as per the PRISMA 2020 statement. The following databases were searched: MEDLINE (inception to 2021); other sources were located via Google Scholar (inception to 2021). The key register used was ClinicalTrials.Gov to locate and identify ongoing or completed trials and identify preliminary or published clinical trial data. Additional entries comprised of records identified from websites (i.e. conference proceedings of ongoing trials), in addition to targeted journal search of the following: New England Journal of Medicine, The Lancet, JAMA, and The BMJ. We systematically searched PubMed from the inception of the database to May 20, 2021, for clinical trials of immune checkpoint inhibitors (atezolizumab and pembrolizumab) approved in triplenegative breast cancer (TNBC). The search terms used across databases are enlisted in Table 1. We restricted the language to English.

Table 1. Search terms.

\begin{tabular}{l}
\hline Search Terms and Combinations Used Across Databases1 \\
\hline (atezolizumab OR pembrolizumab) AND ("triple negative") AND (breast) AND (cancer) \\
("atezolizumab"[Supplementary Concept] OR "atezolizumab"[All Fields] OR ("pembroli- \\
zumab"[Supplementary Concept] OR "pembrolizumab"[All Fields])) AND "triple nega- \\
tive"[All Fields] AND ("breast"[MeSH Terms] OR "breast"[All Fields] OR "breasts"[All \\
Fields] OR "breast s"[All Fields]) AND ("cancer s"[All Fields] OR "cancerated"[All Fields] \\
OR "canceration"[All Fields] OR "cancerization"[All Fields] OR "cancerized"[All Fields] \\
OR "cancerous"[All Fields] OR "neoplasms"[MeSH Terms] OR "neoplasms"[All Fields] OR \\
"cancer"[All Fields] OR "cancers"[All Fields]) \\
${ }^{1}$ A combination of these were used across registers and other sources.
\end{tabular}

\subsection{Selection Process}

The titles and abstracts of identified studies in addition to register and other source records were independently screened by two reviewers (MA and SS) and were doublechecked by a third and fourth reviewer (ZS and AS). The secondary review of full papers was undertaken by the third and fourth reviewers (ZS and AS) and the Cohen's Coefficient of Agreement was calculated to quantify inter-reviewer agreement.

\subsection{Data analysis}

Bibliographic entries from all identified database records were stored in the Endnote software (X9, Clarivate Analytics, USA). Any duplicates were removed using the Endnote deduplication tool. No duplicates were found, neither removed from additional sources (i.e. websites, ClinicalTrials.Gov). A quantitative analytical methodology was applied, to ascertain differences in efficacy outcomes post adjuvant and neoadjuvant treatment, with an analysis of PDL1+ subgroups conducted. For continuous variables, namely PFS and OS, the standardized mean difference (SMD), reported as Cohen's d, with 95\% confidence interval (CI) was computed using a fixed-effects model. We presented pooled estimates, 95\% CI, Odds Ratio (OR) for dichotomous data, namely ORR and pCR, using the randomeffects model. Forest plots documenting OR, SMD, 95\% CI are presented through Figures 2-5. The minimum requirement to assess meta-analysis findings using plots was at least two or more trials reporting the same outcome measure. While a funnel plot was not generated to assess publication bias due to the limited number of trials, (less than 10), the 
heterogeneity between included studies was assessed using the I2 index and the $\chi^{2}$-based $\mathrm{Q}$ test. All statistical analyses were conducted using Review Manager (RevMan) 5.4.

\section{Results}

Figure 1 illustrates the PRISMA flowchart for this meta-analysis. The overall Kappa score computed for the inter-rater agreement was 0.92 indicating an almost perfect agreement. During the first round of screening databases and registered, a total of 215 records were identified; identification of studies via other methods yielded 264 records. From all sources, 440 records were retrieved, of which 48 were assessed for eligibility. Finally, 6 trials were included in the meta-analysis.

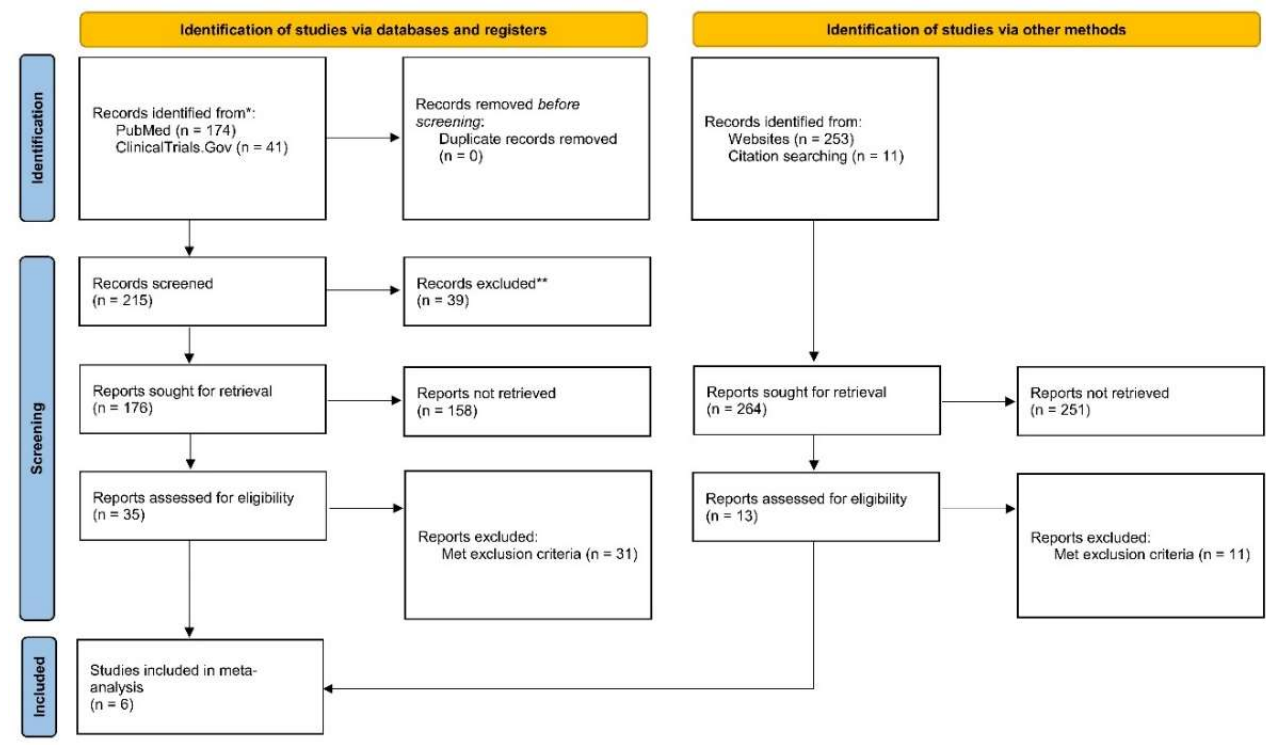

Figure 1. PRISMA flowchart.

\subsection{Outcomes in Adjuvant Therapeutic Trials}

\subsubsection{ORR in Atezolizumab/Pembrolizumab Plus Chemotherapy vs Placebo With PDL1+ Sugroup Analysis}

Three of the 3-adjuvant therapeutic trials presented data of ORR upon treatment with Atezolizumab/Pembrolizumab plus chemotherapy vs placebo. We found that patients in the treatment arm (Atezolizumab/Pembrolizumab plus chemotherapy) had higher odds of achieving an objective response rate (ORR) as compared to the control groups in the study. The statistical significance was noteworthy $(\mathrm{p}<0.001)$ with higher probabilities of increased ORR in treatment arms (OR=1.35, 95\% CI: 1.14-1.60). There was no heterogeneity between the included studies $\left(\mathrm{I}^{2}=0 \%\right.$ ) (Figure $\left.2 \mathrm{a}\right)$. A sensitivity analysis was conducted to assess these preliminary findings. On removing IMPassion130, the trial with the highest weight $(41 \%)$, the test for overall effect was significant $(\mathrm{OR}=1.26,95 \% \mathrm{CI}: 1.01,1.57, \mathrm{P}=$ 0.04 ) with no heterogeneity in the included studies $\left(\mathrm{I}^{2}=0 \%\right.$ ) (Figure $\left.2 \mathrm{a}\right)$.

Two of the 3-adjuvant trials presented subgroup data of PDL1+ patients on treatment with Atezolizumab plus chemotherapy vs placebo. The findings were pertinent as PDL1+ patient groups also presented with higher odds of increased ORR as compared to the control groups of the included trials. As compared to the larger ITT vs control groups, the PDL1+ subset versus control groups had a higher chance of increased ORR (OR=1.7, 95\% CI: 1.24- 
2.33). The results were statistically significant $(\mathrm{P}=0.001)$, with no heterogeneity among the included studies $\left(\mathrm{I}^{2}=0 \%\right)$ (Figure $\left.2 \mathrm{~b}\right)$.

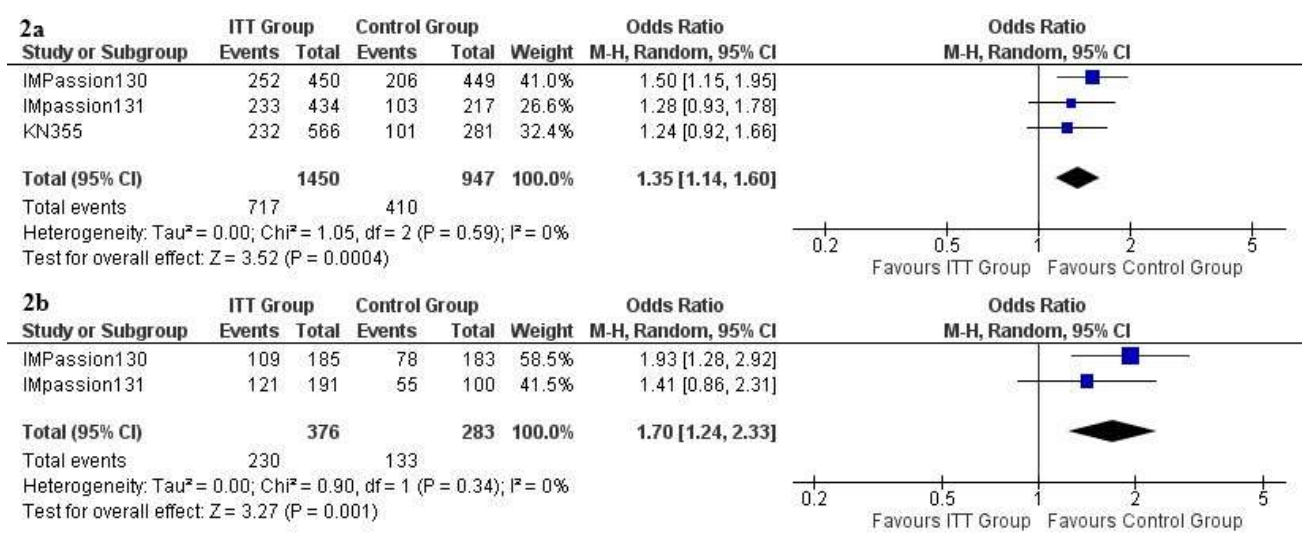

Figure 2. Forest plots for the objective response rate (ORR) in Atezolizumab/Pembrolizumab plus chemotherapy vs placebo with PDL1+ subgroup analysis. (a) The plot illustrates ORR outcomes in three adjuvant trials in the entire group, namely 1) IMpassion130, 2) IMpassion 131 [Atezolizumab plus chemotherapy and vs placebo], and 3) KN355 [Pembrolizumab + Chemotherapy vs placebo]; (b) The plot illustrates ORR outcomes in the subgroup PDL1+, in two adjuvant trials, 1) Impassion131, 2) Impassion 131 [Atezolizumab plus chemotherapy and vs placebo].

\subsubsection{PFS in Atezolizumab plus Chemotherapy vs Placebo with PDL1+ Subgroup Anal- ysis}

All values of SMDs greater than zero indicated the degree to which adjuvant treatment was more efficacious than control groups. Two of the 3 adjuvant studies reported PFS values in the ITT vs Control groups (Atezolizumab plus chemotherapy vs placebo) (Figure 3a). A positive effect size was found for PFS in the ITT arms (Cohen's $d=1.55,95 \%$ CI = $1.40-1.70, \mathrm{P}<0.001)$. Given the diversity of included trials, high heterogeneity was noted $\left(\mathrm{I}^{2}=100 \%\right.$ ) (Figure 3a).

Two of the 3 studies adjuvant studies noted outcomes in the PDL1+ group (ITT vs Control groups). The Atezolizumab plus chemotherapy PDL1+ subgroup had a positive effect size for PFS (Cohen's $d=1.89,95 \% \mathrm{CI}=1.66-2.11, \mathrm{P}<0.001$ ). High heterogeneity was noted in the included studies (100\%) (Figure 3b).

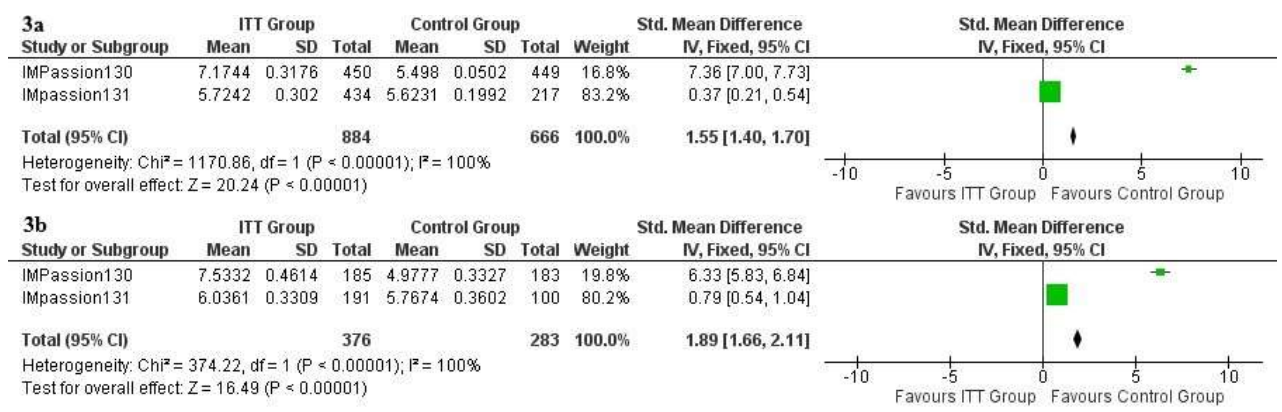

Figure 3. Forest plots for the progression free survival (PFS) in Atezolizumab plus chemotherapy (ITT) vs placebo groups with PDL1+ subgroup analysis; (a) The plot illustrates PFS outcomes in two adjuvant trials in the entire group, namely 1) IMpassion130, 2) IMpassion 131 [Atezolizumab plus chemotherapy and vs placebo]; (b) The plot illustrates PFS outcomes in the subgroup PDL1+, in two adjuvant trials, 1) IMpassion131, 2) IMpassion 131 [Atezolizumab plus chemotherapy and vs placebo]. 


\subsubsection{OS in Atezolizumab plus Chemotherapy vs Placebo}

Of all, only two trials published OS trends in ITT and Control groups. The Atezolizumab plus chemotherapy group had a positive effect size with statistically significant findings as compared to the control groups (Cohen's $\mathrm{d}=0.52,95 \% \mathrm{CI}=0.36-0.68, \mathrm{P}<0.001$ ) (Figure 4). The $\mathrm{I}^{2}$ index was $100 \%$ suggesting high heterogeneity among two of the 3 included studies.

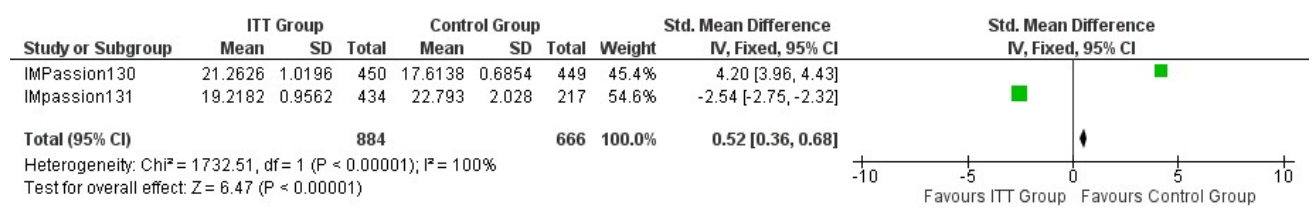

Figure 4. Forest plot for the overall survival (OS) in Atezolizumab plus chemotherapy (ITT) vs placebo groups. The plot illustrates OS outcomes in two trials namely 1) IMpassion130, 2) IMpassion 131 [Atezolizumab plus chemotherapy and vs placebo].

\subsection{Outcome in Neoadjuvant trials}

\subsection{1. pCR Rates in Atezolizumab/Pembrolizumab Plus Chemotherapy vs Placebo with PDL1+ Subgroup Analysis}

Three of the 3 neo-adjuvant trials reported pCR rates in the ITT versus Control groups. We found that patients in the treatment arms had higher odds of achieving pCR as compared to the control groups $(\mathrm{OR}=1.61,95 \% \mathrm{CI}$ : 1.21-2.15). This indicated that neoadjuvant treatment groups had a higher likelihood of obtaining increased pCR rates, ultimately leading to the absence of invasive/in situ cancer in the breast and/or axillary lymph nodes. Achieving pCR was a common outcome measure following neoadjuvant chemotherapy, ultimately leading to improved survival; we found statistical associations to our findings $(\mathrm{P}=0.001)$ (Figure 5a).

Two of the three neo-adjuvant trials reported pCR rates of PDL1+ patients in the ITT versus Control groups. The treatment group had a higher likelihood of presenting with increased pCR rates, ultimately suggesting increased survival, as compared to control groups $(\mathrm{OR}=1.92,95 \% \mathrm{CI}: 1.38-2.68)$. The results were statistically significant $(\mathrm{P}=0.001)$, with no heterogeneity among the included studies $\left(\mathrm{I}^{2}=0 \%\right)$ (Figure $\left.5 \mathrm{~b}\right)$.

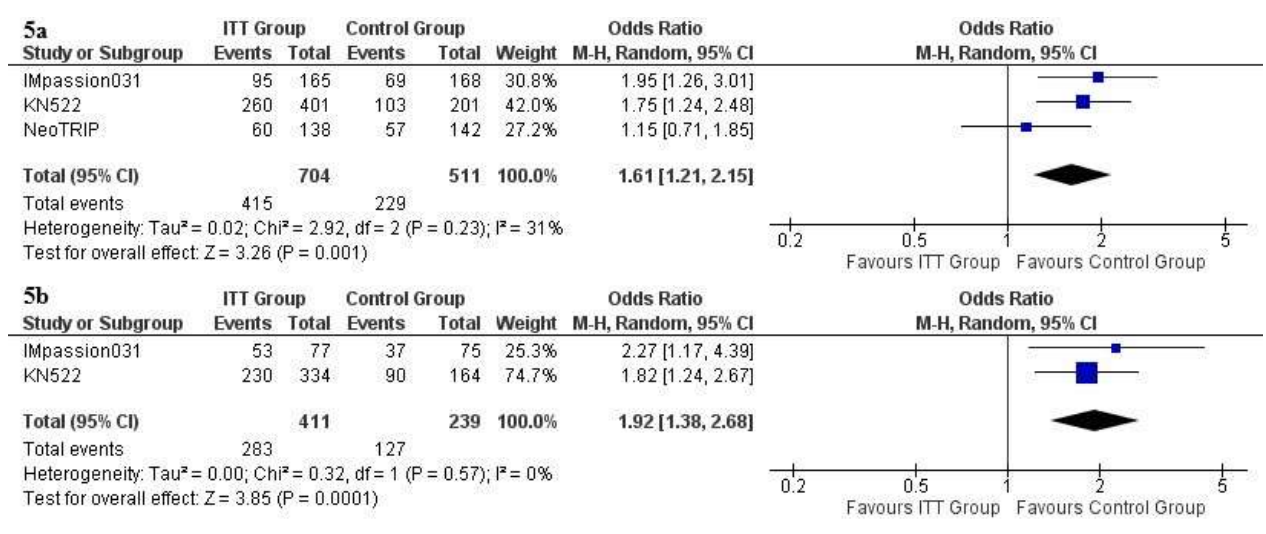

Figure 5. Forest plot for $\mathrm{pCR}$ rates in Atezolizumab/Pembrolizumab plus chemotherapy (ITT) vs placebo groups; (a) The plot illustrates dichotomous pCR outcomes in three trials namely 1) IM- 
passion031, 2) NeoTRIP [Atezolizumab plus chemotherapy and vs placebo] and 3) KN522 [Pembrolizumab + Chemotherapy vs placebo]; (b) The plot illustrates dichotomous pCR outcomes for PDL1+ subgroups in two trials namely 1) IMpassion031 [Atezolizumab plus chemotherapy and vs placebo] and 2) KN522 [Pembrolizumab + Chemotherapy vs placebo].

\section{Discussion}

To our best understanding, this is the first meta-analysis to quantify the efficacy of immune checkpoint inhibitors atezolizumab and pembrolizumab in adjuvant and neoadjuvant settings among patients with TNBC. We reported six clinical trials of pembrolizumab (KN355 and KN522) and atezolizumab (IMpassion130, IMpassion131, Neotrip and IMpassion031) in our pooled meta-analysis. The trials provided atezolizumab and pembrolizumab as either first-line or beyond, and either adjuvant or neoadjuvant settings among patients with TNBC. Our findings demonstrate stronger comparable efficacy among PDL1+ subgroups (IMpassion130 and IMpassion131) in adjuvant settings with either atezolizumab or pembrolizumab in combination with chemotherapy. In the overall group in adjuvant setting, IMpassion130, IMpassion131 and KN355 demonstrated positive efficacy as well. In the neoadjuvant setting, a similar trend was observed in PDL1+ subgroups (IMpassion031 and KN522); the overall efficacy was also positive among all the neoadjuvant trials (IMpassion031, KN522 and Neotrip). The most common immunemediated adverse event was hypothyroidism in $4-18 \%$ of the intention-to-treat patients across the clinical trials.

In adjuvant settings, IMpassion 130 and IMpassion131 were both conducted in firstline settings yet IMpassion131 failed to validate the improved outcomes of atezolizumab and nab-paclitaxel of IMpassion130. Potentially, the findings of IMpassion131 trial [8] were negative and different as compared to the IMpassion130 trial [9] despite having similar designs due to the use of paclitaxel rather than nab-paclitaxel that was used in the early IMpassion130 trial as well as the higher use of steroids. Both atezolizumab and pembrolizumab have been observed to be well tolerated. Concerning current practices, it is pertinent to identify biomarkers when deciding the best treatment regiments for patients with TNBC [10]. During planning TNBC care, the following features that may improve efficacy on treating with immune checkpoint inhibitors ought to be prioritized, including higher PD-L1 expression [11], higher mutational load [12], and increased tumor-infiltrating lymphocyte levels (TILs) [13].

Overall, combination regiments of PD-1/L1 inhibitors have demonstrated efficacious outcomes among patients with TNBC who have already received treatment as well as among treatment-naïve patients in both metastatic and early-stage TNBC. The health-related quality of life (HRQoL) was documented during the treatment and followed every 4 weeks during the 1 year follow-up in the IMpassion130 trial [14]. There were no comparable differences in the HRQoL between the intention-to-treat patients and the placebo group receiving atezolizumab and nab-paclitaxel as first-line for metastatic TNBC [14]. The challenge of treating metastatic TNBC remains in closely monitoring the most critical endpoint including survival as well as symptom relief and quality of life, which is a primary concern in care provision and trials.

The development of new targeted drugs in oncological require careful designing of phase II/III trials. These trials need to be powered to assess for OS, conducted until the outcomes are mature, and carefully monitored for radiological evaluation by independent reviewers [15]. As has been observed across clinical trials for TNBC, an exploration of biomarkers will allow for a better treatment protocol and selection of patients. Further studies have commenced reviewing the enhancement of efficacy in neo-adjuvant and ad- 
juvant settings and with different combination regimens. While the outcomes of the clinical trials have been promising, results ought to be reviewed with extended follow-up data, especially monitoring long-term immune-related toxicity [16].

Despite the approval of atezolizumab in combination with nab-paclitaxel and pembrolizumab in combination with chemotherapy in PD-L1 positive locally-advanced and metastatic TNBC, there are still various aspects of immunotherapy that need to be addressed in TNBC care. The synergistic role of chemotherapy and immunotherapy cannot be undermined given the contrasting outcomes of the IMpassion130 and IMpassion131 trials. Therapies have not been catered to patients for PD-L1 negative TNBC or TNBC refractory to anti-PD-L1 immunotherapy. Clinical trials focusing on mechanisms of response and resistance to immunotherapy are compulsory to develop and advance the outcomes of immunotherapy in TNBC.

\section{Limitations}

Our findings must be used with caution given the limitations in obtaining efficacy and safety data from ongoing trials. It ought to be acknowledged that a diverse racial and ethnic population was not present in all included trials, hence, limiting the applicability to all racial groups. In addition, KN355, the metastatic adjuvant Pembrolizumab and chemotherapy trial did not present data so far for progression free survival or overall survival. The metastatic adjuvant Atezolizumab and chemotherapy trials, IMPassion130 and IMpassion131, published non-estimated interquartile ranges for PDL1+ subgroups for overall survival. The metastatic neoadjuvant Atezolizumab and chemotherapy trial, NeoTRIP, had unquantified PDL1+ subgroups to assess pCR outcomes. Finally, on requesting additional data from the principle investigators in the included trials, no response was received.

\section{Conclusions}

Our findings collate and synthesize better efficacy using markers including pCR in neoadjuvant settings and ORR, PFS, and OS in adjuvant settings with atezolizumab and pembrolizumab. Our analysis offers insight to the outcomes of atezolizumab or pembrolizumab with combination chemotherapy with chemotherapeutic agents. Following the approval of the immunotherapeutic drugs for metastatic TNBC in combination with chemotherapy, ongoing clinical trials are focusing on efficacy outcomes in different subgroups of patients. More insight into the efficacy, and adverse events may help determine and optimize treatment regimens for patients with TNBC.

Author Contributions: Conceptualization, Azza Sarfraz and Zouina Sarfraz; Data curation, Azza Sarfraz and Zouina Sarfraz; Formal analysis, Mahwish Amin, Waleed Asghar, Humaira Sadaf and Azza Sarfraz; Funding acquisition, Shaiza Sharif and Ivan Cherrez-Ojeda; Investigation, Mahwish Amin, Shaiza Sharif and Waleed Asghar; Methodology, Mahwish Amin, Shaiza Sharif, Waleed Asghar and Humaira Sadaf; Project administration, Ivan Cherrez-Ojeda; Resources, Azza Sarfraz and Zouina Sarfraz; Software, Mahwish Amin, Shaiza Sharif, Waleed Asghar and Zouina Sarfraz; Visualization, Waleed Asghar, Humaira Sadaf, Azza Sarfraz and Zouina Sarfraz; Writing - original draft, Mahwish Amin, Shaiza Sharif, Waleed Asghar, Humaira Sadaf, Azza Sarfraz and Zouina Sarfraz; Writing - review \& editing, Shaiza Sharif. All authors have read and agreed to the published version of the manuscript.

Funding: This research received no external funding.

Institutional Review Board Statement: Not applicable.

Conflicts of Interest: The authors declare no conflict of interest. 


\section{References}

1. Anders C, Carey LA. Understanding and treating triple-negative breast cancer. Oncology (Williston Park). 2008, 22, $1233-1243$. 2. Isakoff SJ. Triple-negative breast cancer: role of specific chemotherapy agents. Cancer J, 2010, 16, 53-61. doi:10.1097/PPO.0b013e3181d24ff7

3. Ostrand-Rosenberg S, Horn LA, Haile ST. The programmed death-1 immune-suppressive pathway: barrier to antitumor immunity. J Immunol, 2014, 193, 3835-3841. doi:10.4049/jimmunol.1401572

4. FDA. FDA approves atezolizumab for PD-L1 positive unresectable locally advanced or metastatic triple-negative breast cancer. Available online: https://www.fda.gov/drugs/drug-approvals-and-databases/fda-approves-atezolizumab-pd-l1-positiveunresectable-locally-advanced-or-metastatic-triple-negative (accessed on 20 May 2021).

5. FDA. FDA grants accelerated approval to pembrolizumab for locally recurrent unresectable or metastatic triple negative breast cancer. Available online: https://www.fda.gov/drugs/drug-approvals-and-databases/fda-grants-accelerated-approvalpembrolizumab-locally-recurrent-unresectable-or-metastatic-triple (accessed on 20 May 2021).

6. Lipson EJ, Forde PM, Hammers H-J, Emens LA, Taube JM, Topalian SL. Antagonists of PD-1 and PD-L1 in Cancer Treatment. Semin Oncol, 2015, 42, 587-600. doi:10.1053/j.seminoncol.2015.05.013

7. Page MJ, McKenzie JE, Bossuyt PM, et al. The PRISMA 2020 statement: An updated guideline for reporting systematic reviews. BMJ, 2021. doi:10.1136/bmj.n71

8. Miles D, Andre F, Gligorov J, et al. IMpassion131: Phase III study comparing 1L atezolizumab with paclitaxel vs placebo with paclitaxel in treatment-naive patients with inoperable locally advanced or metastatic triple negative breast cancer (mTNBC). Ann Oncol, 2017, 28, v105. doi:10.1093/annonc/mdx365.080

9. Schmid P, Adams S, Rugo HS, et al. Atezolizumab and Nab-Paclitaxel in Advanced Triple-Negative Breast Cancer. N Engl J Med. 2018, 379, 2108-2121. doi:10.1056/NEJMoa1809615

10. Palleschi M, Maltoni R, Sarti S, Melegari E, Bravaccini S, Rocca A. Immunotherapy: The end of the "dark age" for metastatic triple-negative breast cancer? Breast J, 2020, 26, 739-742. doi:https://doi.org/10.1111/tbj.13662

11. Cerbelli B, Pernazza A, Botticelli A, et al. PD-L1 Expression in TNBC: A Predictive Biomarker of Response to Neoadjuvant Chemotherapy? Gasparri ML, ed. Biomed Res Int, 2017, 1750925. doi:10.1155/2017/1750925

12. Budczies J, Bockmayr M, Denkert C, et al. Classical pathology and mutational load of breast cancer - integration of two worlds. J Pathol Clin Res, 2015, 1, 225-238. doi:https://doi.org/10.1002/cjp2.25

13. Beckers RK, Selinger CI, Vilain R, et al. Programmed death ligand 1 expression in triple-negative breast cancer is associated with tumour-infiltrating lymphocytes and improved outcome. Histopathology, 2016, 69, 25-34. doi:https://doi.org/10.1111/his.12904

14. Adams S, Diéras V, Barrios CH, et al. Patient-reported outcomes from the phase III IMpassion130 trial of atezolizumab plus nab-paclitaxel in metastatic triple-negative breast cancer. Ann Oncol, 2020, 31, 582-589. doi:https://doi.org/10.1016/j.annonc.2020.02.003

15. Lyman GH, Burstein HJ, Buzdar AU, D’Agostino R, Ellis PA. Making Genuine Progress Against Metastatic Breast Cancer. J Clin Oncol, 2012, 30, 3448-3451. doi:10.1200/JCO.2012.43.6931

16. Keenan TE TS. Role of Immunotherapy in Triple-Negative Breast Cancer. J Natl Compr Canc Netw. 2020, 18, 479-489. doi:10.6004/jnccn.2020.7554 